\title{
Proceedings of the second international workshop on Code OptimiSation for Multl and many Cores COSMIC
}

\section{San Francisco CA, USA February 8, 2015}

\author{
held in conjunction with the International \\ Symposium on Code Generation and Optimization \\ (CGO) 2015
}

\section{Program Co-Chairs:}

Zheng Wang, Lancaster University

Pavlos Petoumenos, The University of Edinburgh Hugh Leather, The University of Edinburgh 


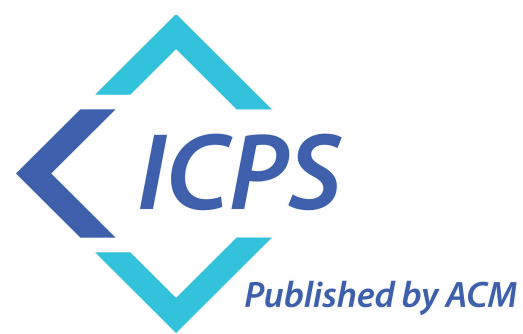

The Association for Computing Machinery

2 Penn Plaza, Suite 701

New York New York 10121-0701

ACM COPYRIGHT NOTICE. Copyright $\odot 2015$ by the Association for Computing Machinery, Inc. Permission to make digital or hard copies of part or all of this work for personal or classroom use is granted without fee provided that copies are not made or distributed for profit or commercial advantage and that copies bear this notice and the full citation on the first page. Copyrights for components of this work owned by others than ACM must be honored. Abstracting with credit is permitted. To copy otherwise,

to republish, to post on servers, or to redistribute to lists, requires prior specific permission and/or a fee. Request permissions from Publications Dept., ACM, Inc., fax +1 (212) 869-0481, or permissions@acm.org.

For other copying of articles that carry a code at the bottom of the first or last page, copying is permitted provided that the per-copy fee indicated in the code is paid through the Copyright Clearance Center, 222 Rosewood Drive, Danvers, MA 01923, +1-978-750-

$$
\text { 8400, +1-978-750-4470 (fax). }
$$

\section{Notice to Past Authors of ACM-Published Articles}

ACM intends to create a complete electronic archive of all articles and/or other material previously published by ACM. If you have written a work that was previously published by ACM in any journal or conference proceedings prior to 1978, or any SIG Newsletter at any time, and you do NOT want this work to appear in the ACM Digital Library, please inform permissions@acm.org, stating the title of the work, the author(s), and where and when published.

ACM ISBN: 978-1-4503-3316-0 


\section{Table of Contents}

\section{Session I}

Compilers and Tools

- Dependence-Based Code

Transformation for Coarse-Grained Parallelism

Article 1

Bo Zhao, Zhen Li, Ali Jannesari, Felix Wolf and Weiguo Wui

- Hardware-Aware Automatic Code

Transformation to Support Compilers in Exploiting

the Multi-Level Parallel Potential of Modern CPUs.

Article 2

Dustin Feld, Thomas Soddemann, Sven Mallach and Michael Juenger

- The Basic Building Blocks of Parallel Tasks..........................Article 3

Rohit Atre, Ali Jannesari and Felix Wolf

\section{Session II}

GPUs and Accelerators

- Runtime Support for Multiple Offload-Based

Programming Models on Embedded Manycore.

Article 4

Alessandro Capotondi, Germain Haugou, Andrea Marongiu and

Luca Benini

- Exploiting Dynamic Parallelism to

Efficiently Support Irregular Nested Loops on GPUs.........Article 5

$\mathrm{Da}$ Li, Hancheng Wu and Michela Becchi

- An Evaluation of Memory Sharing

Performance for Heterogeneous

Embedded SoCs with Many-Core Accelerators

Article 6

Pirmin Vogel, Andrea Marongiu and Luca Benini

\section{Session III}

Models and Paradigms

- A Roadmap for a Type Architecture

Based Parallel Programming Language.

Article 7

Muhammad Nur Yanhaona and Andrew Grimshaw

- Cycle-based Model to Evaluate Consistency Protocols within a Multi-protocol Compilation Toolchain. Article 8

Safae Dahmani, Hamza Chaker, Loïc Cudennec, Guy Gogniat, and Martha Johanna Sepulveda 